\title{
The efficacy and safety of intrathecal dexmedetomidine for parturients undergoing cesarean section: a double- blind randomized controlled trial
}

\author{
Xiao-xiao $\mathrm{Li}^{1 \dagger}$, Yu-mei Li ${ }^{2 \dagger}$, Xue-li Lv ${ }^{1}$, Xing-he Wang ${ }^{1}$ and Su Liur, ${ }^{1,3^{*}}$
}

\begin{abstract}
Background: The efficacy and safety of spinal anesthesia by intrathecal dexmedetomidine (DEX) for parturients undergoing cesarean section are still lack of evidence. This aim of our study was to evaluate the efficacy and safety of intrathecal DEX for parturients undergoing cesarean section to provide more data evidence for intrathecal applications.

Methods: Three hundred parturients undergoing cesarean section under spinal anesthesia were randomly assigned into three groups: group B: $9.0 \mathrm{mg}(1.2 \mathrm{ml})$ of $0.75 \%$ bupivacaine with saline $(1 \mathrm{ml})$; group FB: $9.0 \mathrm{mg}(1.2 \mathrm{ml})$ of $0.75 \%$ bupivacaine with $20 \mu \mathrm{g}$ of fentanyl $(1 \mathrm{ml})$; group DB: $9.0 \mathrm{mg}(1.2 \mathrm{ml})$ of $0.75 \%$ bupivacaine with $5 \mu \mathrm{g}$ of DEX $(1 \mathrm{ml})$. Intraoperative block characteristics, parturients' postoperative quality of recovery, maternal and neonatal outcomes and the plasma concentration of DEX were measured. All parturients were followed up for 30 days to determine whether nerve injury occurred.
\end{abstract}

Results: Compared with group B, the duration of sensory block in group FB and group DB were significantly prolonged (108.4 min [95\% Confidence Interval $(C l)=104.6-112.3$ in group $B$, and 122.0 min $[95 \% \mathrm{Cl}=116.8-127.3]$ in group $\mathrm{FB}, 148.2 \mathrm{~min}[95 \% \mathrm{Cl}=145.3-151.1]$ in group DB). The overall score of quality recovery in group DB (71.6 $[95 \% \mathrm{Cl}=71.0-72.2])$ was significantly higher than that in group FB $(61.5[95 \% \mathrm{Cl}=60.8-62.2])$ and group $\mathrm{B}(61.7$ $[95 \% \mathrm{Cl}=61.0-62.4])$. There was no statistically significant difference among the three groups for $\mathrm{PH}, \mathrm{PaO}_{2}$, and $\mathrm{PaCO}_{2}$ of newborn. The plasma concentration of DEX in umbilical artery and umbilical vein was low and cannot be detected. The 30-days follow-up of parturients did not show any new onset of back, buttock or leg pain or paresthesia.

Conclusions: DEX is a potential local anesthetic adjuvant that the intrathecal combination of $5 \mu \mathrm{g}$ DEX can safely exhibit a facilitatory block effect and improve parturients' recovery quality.

(Continued on next page)

\footnotetext{
* Correspondence: liusu112277@gmail.com

${ }^{+}$Xiao-xiao Li and Yu-mei Li contributed equally to this work.

'Jiangsu Province Key Laboratory of Anesthesiology, Xuzhou Medical University, Xuzhou, Jiangsu, China

${ }^{3}$ Department of Anesthesiology, the Affiliated Hospital of Xuzhou Medical University, 99 Huaihai West Road, Xuzhou 221000, Jiangsu, China Full list of author information is available at the end of the article
}

(c) The Author(s). 2020 Open Access This article is licensed under a Creative Commons Attribution 4.0 International License, which permits use, sharing, adaptation, distribution and reproduction in any medium or format, as long as you give appropriate credit to the original author(s) and the source, provide a link to the Creative Commons licence, and indicate if changes were made. The images or other third party material in this article are included in the article's Creative Commons licence, unless indicated otherwise in a credit line to the material. If material is not included in the article's Creative Commons licence and your intended use is not permitted by statutory regulation or exceeds the permitted use, you will need to obtain permission directly from the copyright holder. To view a copy of this licence, visit http://creativecommons.org/licenses/by/4.0/. The Creative Commons Public Domain Dedication waiver (http://creativecommons.org/publicdomain/zero/1.0/) applies to the data made available in this article, unless otherwise stated in a credit line to the data. 
(Continued from previous page)

Trial registration: Chinese Clinical Trial Registry (Registration number \# ChiCTR1900022019; Date of Registration on March 20th, 2019).

Keywords: Intrathecal dexmedetomidine, Spinal anesthesia, Cesarean section

\section{Background}

Spinal anesthesia, with the advantage of easy-operating and avoiding the maternal risk of general anesthesia, including tracheal intubation failure, aspiration and lung infection, has been recommended as the preferred anesthesia for cesarean section [1-4]. However, some disadvantages caused by single-shot spinal anesthesia such us the limited duration of action and insufficient postoperative analgesia, which will lower the maternal postoperative recovery quality, and increasing local anesthetics doses is prone to cause maternal and neonatal adverse events $[5,6]$. Therefore, several adjuvants $[7,8]$ in combination with local anesthetics have gradually been applicated to further improve spinal anesthesia, of which dexmedetomidine (DEX) is a good choice.

DEX, a highly selective $\alpha-2$ adrenergic receptor agonist, provides sedative, analgesic, anti-sympathetic effects and has no significant effect on respiration [9]. Several clinical trials [10-13] have shown that DEX can be applicated as an auxiliary for spinal anesthesia through enhancing the anesthetic effects, preventing and reducing adverse reactions caused by local anesthetics. However, there are only a few studies on intrathecal DEX for cesarean section and these studies were mostly singlecenter with a small sample size, and whether the parturients' recovery quality would be improved and whether DEX would adversely affect the fetus are still lack of plasma concentration evidence. Therefore, this twocenters, prospective, double-blind, randomized controlled trial was designed to evaluate the efficacy and safety of intrathecal DEX for parturients undergoing cesarean section to provide more data evidence for intrathecal applications.

\section{Methods}

\section{Study participants}

This trial was approved by the ethics committee of Feng Xian People's Hospital and the Affiliated Hospital of Xuzhou Medical University. Written informed consent was obtained from all enrolled participants. This manuscript adheres to the applicable CONSORT guidelines. This study was a two-centers, prospective, double-blind, randomized controlled trial, and the two centers are the Affiliated Hospital of Xuzhou Medical University and Feng Xian People's Hospital. Patient recruitment and data collection were started in April 2019 and ended in July 2019. The inclusion criteria of our study were: (1)
Full-term pregnant women undergoing elective cesarean section under spinal anesthesia; (2) Age: $20 \sim 35$ years; (3) ASA physical status II III; The exclusion criteria were: (1) Multiple pregnancies; (2) Cardiovascular disease (e.g., pre-eclampsia and hypertension); (3) Serious hepatic dysfunction (Child-Pugh class C); (4) serious renal dysfunction (undergoing dialysis before surgery); (4) History of alcohol or opioid addiction; (5) Contraindication to spinal anesthesia; (7) Refusing to sign informed consent.

\section{Randomization, blinding and allocation concealment}

According to the random number generated by computer, parturients were randomly allocated into three equal groups to receive either DEX, fentanyl or normal saline in combination with bupivacaine. The randomization sequence was placed in serially numbered opaque envelopes. Before the start of spinal anesthesia, an anesthesiologist prepared relevant drugs according to the randomization sequence and the anesthesiologist would not participate in the data collection, follow-up, and analysis.

\section{Study interventions}

All parturients included in the study routinely fasted for 6-8 h before surgery, and none of them received premedication. When parturients were admitted into the operating room, standard monitoring for pulse oxygen saturation $\left(\mathrm{SpO}_{2}\right)$, heart rate $(\mathrm{HR})$, electrocardiogram (ECG), and noninvasive blood pressure (NIBP) was carried out. All parturients were given a supplementation of $3 \mathrm{~L} / \mathrm{min} \mathrm{O}_{2}$ through the nasal catheter. Then an intravenous 18-G cannula was inserted and patients were preloaded with ringer lactate $10 \mathrm{ml} / \mathrm{kg} 15-20 \mathrm{~min}$ before anesthesia.

With the parturients in the left lateral position, spinal anesthesia was performed at the L3-L4 interspace with a $25 \mathrm{G}$ spinal Quincke-tip needle and study drugs were injected slowly within $15 \mathrm{~s}$ after the cerebrospinal fluid flowing out. The three groups were scheduled to receive drugs as follows: bupivacaine group (group B): $9 \mathrm{mg}(1.2 \mathrm{ml})$ of $0.75 \%$ bupivacaine, with $1.0 \mathrm{ml}$ of normal saline. Bupivacaine + fentanyl group: (group FB): $9 \mathrm{mg}(1.2 \mathrm{ml})$ of $0.75 \%$ bupivacaine, with $20 \mu \mathrm{g}$ of fentanyl in $1.0 \mathrm{ml}$ of normal saline. Bupivacaine + DEX group (group DB): 9 $\mathrm{mg}(1.2 \mathrm{ml})$ of $0.75 \%$ bupivacaine, with $5 \mu \mathrm{g}$ of DEX 
in $1.0 \mathrm{ml}$ of normal saline. After removing the spinal needle, parturients were in the position with a 15degree tilt to the left side immediately. All spinal anesthesia procedures were performed by experienced anesthesiologists. The sensory block level was tested by the pinprick method using a blunt 25-G needle. Assessment of the dermatomal level was done every 1 min until the peak sensory block level was achieved. Subsequently frequent testing every $10 \mathrm{~min}$ was performed until regression to S1 dermatome. The motor block was assessed by the modified Bromage scale (MBS, $0=$ no paralysis, $1=$ inability to raise the leg, $2=$ inability to flex the knee, and $3=$ inability to flex the ankle) [14]. Surgery was allowed to commence once the sensory block level reached T6 [15]. Any patient showing moderate pain (visual analog score $(\mathrm{VAS}) \geq 3$ ) was administered intravenous $0.5 \mathrm{mg} / \mathrm{kg}$ ketamine. If hypotension (systolic blood pressure $(\mathrm{SBP})<90 \mathrm{mmHg}$ or descending baseline values by $30 \%)$ persisted, intravenous $6 \mathrm{mg}$ of ephedrine was administered; If bradycardia ( $\mathrm{HR}<50 \mathrm{bpm})$ occurs, intravenous $0.5 \mathrm{mg}$ of atropine was administered. Repeat if necessary. Intraoperative ephedrine and atropine consumption were recorded. After surgery, all patients underwent patient-controlled intravenous analgesia (PCIA) with $2 \mu \mathrm{g} / \mathrm{kg}$ of sufentanil and $10 \mathrm{mg}$ of tropisetron.

\section{Outcomes}

The primary outcome of our study was the duration of sensory block, which was defined as time taken from intrathecal injection to sensory regression to S1 dermatome. The secondary outcomes of our study were as follows: the onset time of sensory block, which was defined as time taken from intrathecal injection to the maternal feeling of lower extremities temperature increment or numbness [16]; the onset time of motor block, which was defined as time taken from intrathecal injection to MBS > 1; the duration of motor block, which was defined as time taken from intrathecal injection to MBS = 0 ; the peak sensory block level; the blood gas analysis for $\mathrm{PH}, \mathrm{PaO}_{2}$, and $\mathrm{PaCO}_{2}$ of the umbilical artery (UA) and umbilical vein (UV) blood samples of the newborn, which was performed immediately after collection; the plasma concentration of DEX in the UA and UV, which was determined by High-Performance Liquid Chromatography Tandem Mass Spectrometry methods [17]; Apgar scores, which were assessed at 1st and 5 th min by the obstetrician who was blinded to the study; the hemodynamic parameters of parturient including BP, HR, which were evaluated at: baseline values (T0), immediately after blockcade (T1), 5 min (T2), 10 min (T3), $15 \mathrm{~min}$ (T4) and $20 \mathrm{~min}$ (T5) after blockcade. BP and $\mathrm{HR}$ at T0 were defined as the average values measured for 3 consecutive times at rest after entering the operating room.

The recovery quality of parturients within $24 \mathrm{~h}$ after surgery was assessed by obstetric quality of recovery- 11 score [18] (ObsQoR-11, score from 0 to 10 in each term, where $0=$ strongly agree and $10=$ strongly disagree, the higher of the score, the higher of recovery quality), which was designed for parturients and presented by Ciechanowicz S; intra-and postoperative adverse events including nausea, vomiting and shivering, time to the first analgesic request and total sufentanil comsumption at $24 \mathrm{~h}$ after surgery were also recorded. Parturients were contacted by telephone for a post-operative 30 days following discharge to determine whether nerve injury occurred, including any new onset of back, buttock or leg pain or paresthesia. All of these evaluations were performed by an anesthesiologist blind to any other aspect of the trial.

\section{Statistical analysis}

The sample size was calculated using PASS 15.0 software (NCSS, LLC, Kaysville, USA). The sample size calculation was based on the primary outcome, the duration of sensory block. According to our pilot trial results, the duration of sensory block was $114.3 \pm 28.5 \mathrm{~min}$ for group B, $120.1 \pm 29.4 \mathrm{~min}$ for group FB, $128.8 \pm 29.5 \mathrm{~min}$ for group DB. A total of 80 patients were required to achieve $80 \%$ power with an alpha error of $5 \%$ based on the module of analysis of variance (ANOVA) in PASS. Considering a lost-to-follow-up rate of about 15\%, 94 patients are required for each group. Finally, a total of 100 parturients were recruited in our study.

Statistical analysis was performed using IBM SPSS 22.0 software (SPSS Inc., IBM, Chicago, IL, USA). Numeric variables were analyzed for normality by the Kolmogorov-Smirnov test. Normally distributed continuous variables were expressed as mean with a standard deviation and compared using ANOVA with post hoc analysis using Bonferroni test. The categorical variables were presented as number (\%) and compared using Chi-square test or Fischer exact test. Kaplan-Meier curve illustrated the time to first analgesic request and comparisons between groups were conducted with the logrank test. Hemodynamic parameters were compared by repetitive measurement deviation analysis. $P<0.05$ was considered statistically significant.

\section{Results}

Between April and July in 2019, 342 pregnant women at two centers were evaluated for study participation. Of these, eighteen women did not meet the inclusion criteria, twenty women refused to participate, and four women were excluded for other reasons (Fig. 1). Finally, three hundred patients were randomly 1:1:1 divided into 


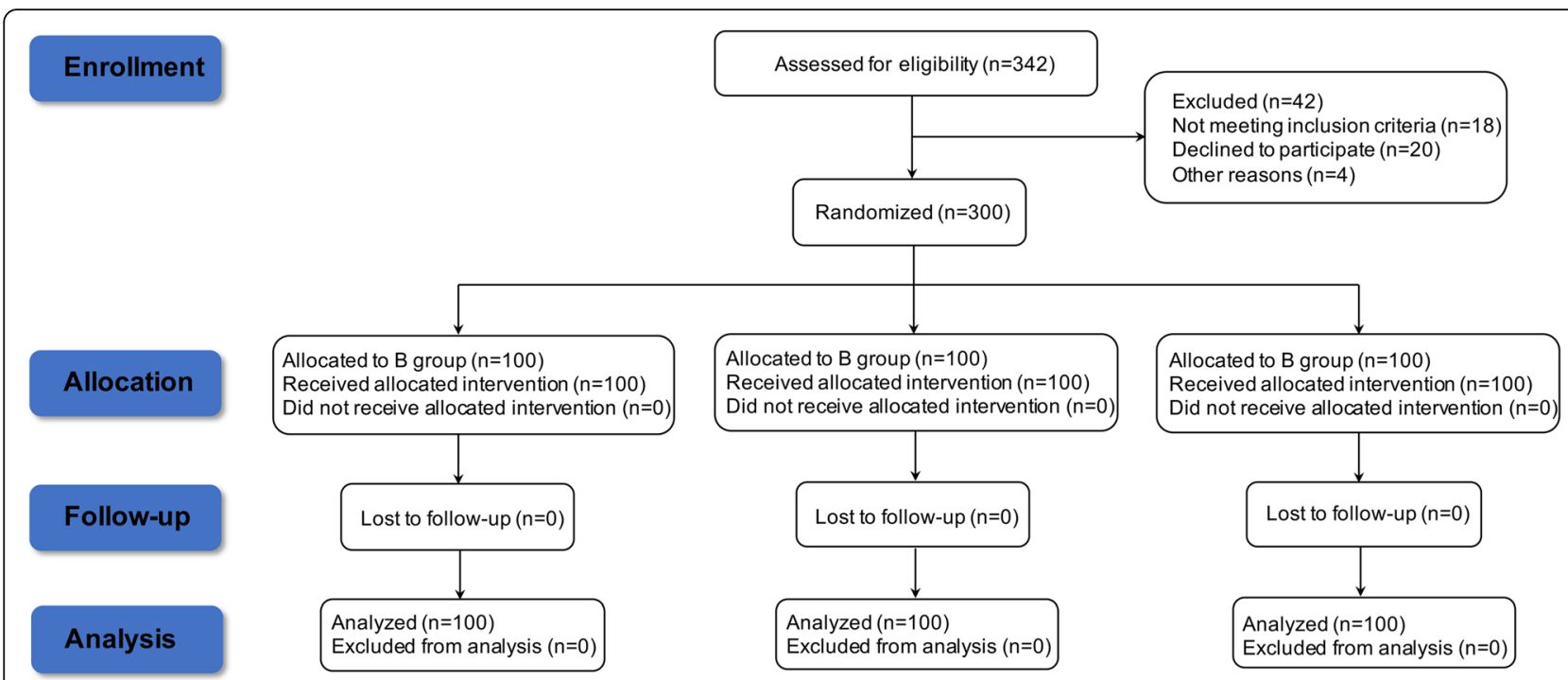

Fig. 1 Study population flow diagram

group B $(n=100)$, group FB $(n=100)$ and group DB $(n=100)$. All patients were well-blocked and no one needed additional analgesia during the surgery. In addition, all patients completed the assessment and received postoperative follow-up for 30 days.

The three groups were comparable with regard to baseline variables include age, height, weight, BMI, ASA physical status, gestational age. There were also no significant differences in perioperative variables including peak sensory level, duration of surgery, intraoperative fluid volume and blood loss (Table 1).
Compared with group B, the duration of sensory block in group FB and group DB were prolonged (108.4 min [95\% Confidence Interval $(\mathrm{CI})=104.6-112.3$ ] in group $\mathrm{B}$, and $122.0 \mathrm{~min}$ [ $95 \% \mathrm{CI}=116.8-127.3]$ in group $\mathrm{FB}$, $148.2 \mathrm{~min}[95 \% \mathrm{CI}=145.3-151.1]$ in group $\mathrm{DB})$ with statistical significance $(P<0.001)$ (Fig. 2a). The duration of sensory block was significantly longer in group DB as compared with group FB $(P<0.001)$. Compared with group $B$, the onset time of sensory block (Fig. 2b) in group DB was significantly shorter $(12.2 \mathrm{~s}[95 \% \mathrm{CI}=$ $12.0-12.4])$ in group $\mathrm{DB}, 14.5 \mathrm{~s}[95 \% \mathrm{CI}=14.0-15.1]$ in

Table 1 Baseline and perioperative characteristics of parturients

\begin{tabular}{|c|c|c|c|c|}
\hline & Group B $(n=100)$ & Group FB $(n=100)$ & Group DB $(n=100)$ & $P$-Value \\
\hline Age (yr.) & $27(26-29)$ & $27(25-30)$ & $27(25-29)$ & 0.244 \\
\hline Height (cm) & 161.5 (159.0-164.0) & $162.0(159.0-165.0)$ & $160(159.0-164.0)$ & 0.284 \\
\hline Weight (kg) & $72.3 \pm 6.2$ & $72.8 \pm 5.3$ & $72.6 \pm 5.3$ & 0.771 \\
\hline $\mathrm{BMI}\left(\mathrm{kg} / \mathrm{m}^{2}\right)$ & $27.6 \pm 2.5$ & $27.6 \pm 2.2$ & $27.8 \pm 2.3$ & 0.865 \\
\hline ASA physical status, n (\%) & & & & 0.394 \\
\hline$\|$ & $78(78)$ & $74(74)$ & $82(82)$ & \\
\hline III & $22(22)$ & $26(26)$ & $18(18)$ & \\
\hline Gestational week (Wk.) & $39(38-39)$ & 39 (38-39) & $39(38-39)$ & 0.379 \\
\hline Peak sensory level & & & & 0.846 \\
\hline $\mathrm{T} 2$ & $2(2)$ & $1(1)$ & $1(1)$ & \\
\hline T4 & $37(37)$ & $36(36)$ & $42(42)$ & \\
\hline T6 & $61(61)$ & $63(63)$ & $57(57)$ & \\
\hline Surgery duration (min) & $41.0(38.0-44.0)$ & $41.0(39.0-44.0)$ & $41.0(39.0-44.0)$ & 0.746 \\
\hline Intraoperative fluid volume (ml) & $1351.1 \pm 115.4$ & $1361.6 \pm 97.8$ & $1356.5 \pm 98.7$ & 0.775 \\
\hline Intraoperative blood loss (ml) & $421.2 \pm 36.0$ & $424.5 \pm 30.5$ & $423.1 \pm 30.7$ & 0.773 \\
\hline
\end{tabular}

Notes: Data are presented as $\mathrm{n}(\%)$ or mean \pm SD or median (range); There were no significant differences among the three groups $(P>0.05)$. Group $\mathrm{B}=$ bupivacaine group; Group FB = bupivacaine and fentanyl group; Group DB = bupivacaine and dexmedetomidine group Abbreviations: ASA American Society of Anesthesiologists, BMI Body Mass Index 

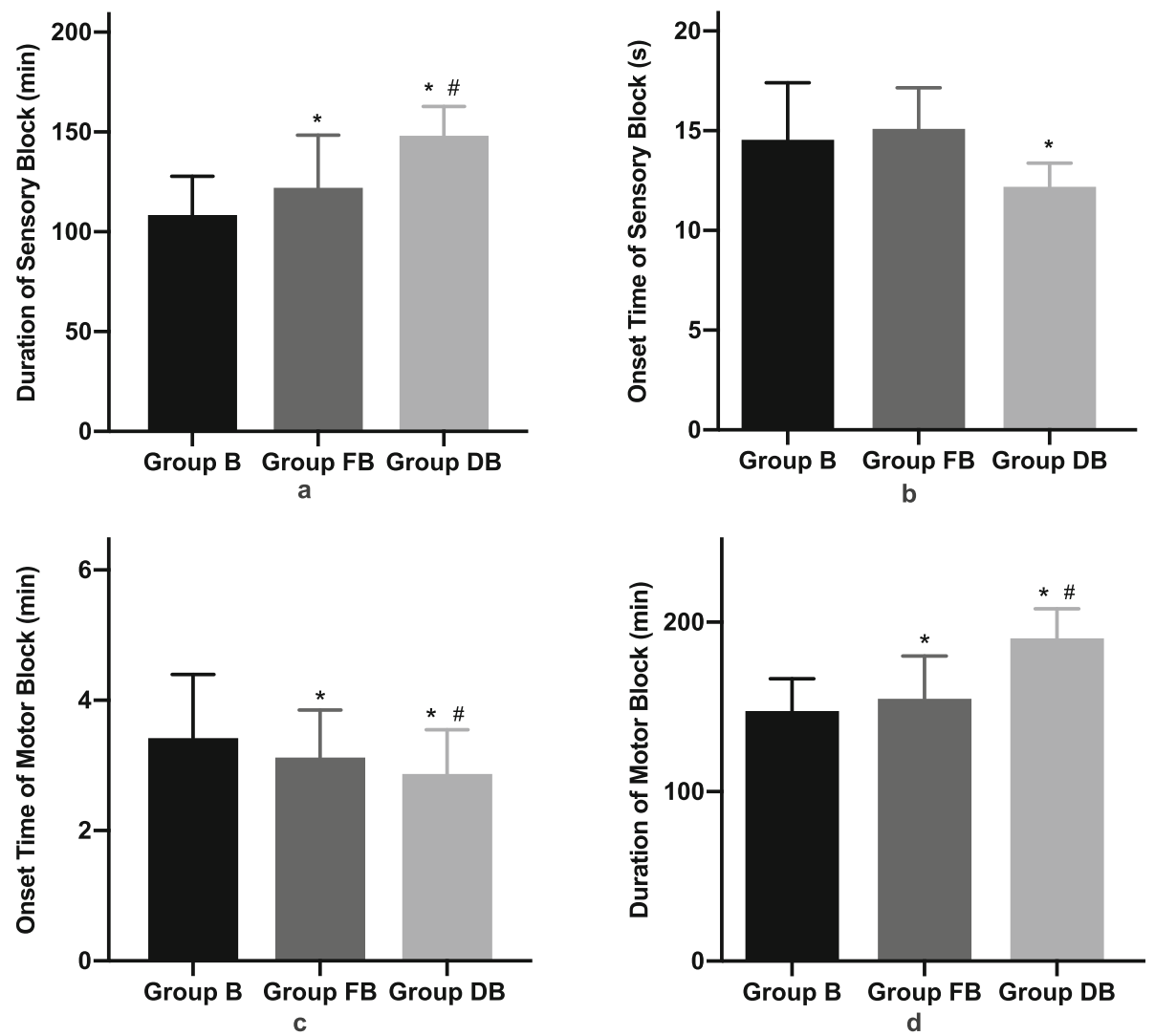

Fig. 2 Block characteristics of parturientsNotes: Group B = bupivacaine group; Group FB = bupivacaine and fentanyl group; Group DB = bupivacaine and dexmedetomidine group. * $P<0.017$ Group DB or Group FB vs Group B; ${ }^{*} P<0.017$ Group DB vs Group FB.

group $\mathrm{B}, P<0.001)$. Besides, compared with group $\mathrm{B}$ and group FB (Fig. 2c), the onset time of motor block in group DB was statistically shorter $(2.9 \mathrm{~min}[95 \% \mathrm{CI}=$ 2.7-3.0] in group $\mathrm{DB}, 3.1 \mathrm{~min}[95 \% \mathrm{CI}=3.0-3.3]$ in group $\mathrm{FB}, 3.4 \mathrm{~min}$ [95\% $\mathrm{CI}=3.2-3.6]$, in group $\mathrm{B}, P<$ $0.001)$. However, compared with group B (147.5 min [95\% CI $=143.7-151.3]$ ), the duration of motor block (Fig. 2d) in group DB (190.3 min [95\% CI =186.9193.8]) was prolonged by $43 \mathrm{~min}(P<0.001)$, while that in group $\mathrm{FB}(154.9 \mathrm{~min}$ [95\% CI $=150.0-160.0])$ was prolonged by $7 \mathrm{~min}(P=0.038)$.

There were 11 items in ObsQoR-11 score Table (Table 2) to reflect the quality of postoperative recovery. The overall score of group DB (71.6 [95\% CI $=71.0-$ $72.2])$ was higher than that of group FB $(61.5$ [95\% CI = 60.8-62.2], $P<0.001)$ and group B (61.7 [95\% CI = 61.062.4], $P<0.001)$. All items showed recovery quality of group DB was significantly better than that of group $B$, except in terms of feeling dizzy $(P>0.05)$. Moreover, there was no statistical difference between group $B$ and group FB about the ObsQoR-11 score.

Kaplan-Meier curve (Fig. 3) showed that time to first analgesic request in group $\mathrm{DB}$ was longer than that in group FB and group B (log-rank $P<0.017)$. However, the sufentanil dosage within postoperative $24 \mathrm{~h}$ was not statistically different among three groups $(P=0.681)$.

The maternal hemodynamic characteristics including $\mathrm{HR}$ and mean arterial pressure (MAP) were found significantly higher in group $\mathrm{DB}$ than that in group $\mathrm{B}$ (Fig. 4). The incidence of shivering (Table 3) was statistically lowered in group DB (3\%) compared with group FB (18\%) and group B (35\%). The incidence of hypotension in group $\mathrm{DB}(33 \%)$ was higher than that in group FB (25\%) and group B (28\%) but with no statistical difference. There was no statistical difference for the dosage of ephedrine and atropine, intra-operative or postoperative nausea and vomiting among three groups.

For $\mathrm{PH}, \mathrm{PaO}_{2}$, and $\mathrm{PaCO}_{2}$ in the umbilical artery and umbilical vein blood of newborn (Table 4), there were no statistically significant among the three groups. The concentration of DEX in umbilical artery and umbilical vein was too low to be detected by High-Performance Liquid Chromatography Tandem Mass Spectrometry. The mean values of Apgar scores at 1st and 5th min were all beyond 8 , which also showed no statistical significance. Moreover, the 30-daysfollow-up did not show any new onset of back, buttock or leg pain or paresthesia. 
Table 2 ObsQoR-11 of parturients

\begin{tabular}{|c|c|c|c|c|}
\hline & Group B $(n=100)$ & Group FB $(n=100)$ & Group DB $(n=100)$ & $P$-Value \\
\hline Moderate pain & $3.6 \pm 1.1$ & $5.6 \pm 0.9^{*}$ & $7.3 \pm 1.2^{* \#}$ & $<0.001$ \\
\hline Severe pain & $4.4 \pm 1.2$ & $5.0 \pm 2.1^{*}$ & $7.5 \pm 1.6^{* \#}$ & $<0.001$ \\
\hline Nausea or vomiting & $5.2 \pm 1.0$ & $6.0 \pm 0.9^{*}$ & $7.3 \pm 1.2^{* \#}$ & $<0.001$ \\
\hline Feeling dizzy & $6.3 \pm 1.2$ & $5.0 \pm 0.8^{*}$ & $6.3 \pm 1.1^{\#}$ & $<0.001$ \\
\hline Shivering & $3.7 \pm 0.8$ & $5.3 \pm 0.7^{*}$ & $7.2 \pm 1.0^{* \#}$ & $<0.001$ \\
\hline Have been comfortable & $6.4 \pm 1.0$ & $6.4 \pm 0.6$ & $7.4 \pm 1.8^{* \#}$ & $<0.001$ \\
\hline Able to mobilize independently & $6.0 \pm 1.9$ & $6.9 \pm 1.5^{*}$ & $6.9 \pm 1.1^{*}$ & $<0.001$ \\
\hline Can hold baby without assistance & $6.9 \pm 0.8$ & $8.2 \pm 0.7^{*}$ & $8.1 \pm 0.8^{*}$ & $<0.001$ \\
\hline Can feed/nurse baby without assistance & $6.6 \pm 0.7$ & $7.0 \pm 1.0^{*}$ & $7.1 \pm 0.7^{*}$ & $<0.001$ \\
\hline Can look after personal hygiene/toilet & $5.6 \pm 0.9$ & $6.3 \pm 0.6^{*}$ & $6.5 \pm 0.9^{*}$ & $<0.001$ \\
\hline Feeling in control & $7.0 \pm 0.8$ & $7.3 \pm 1.0^{*}$ & $8.0 \pm 0.8^{* \#}$ & $<0.001$ \\
\hline Total & $61.7 \pm 3.3$ & $61.5 \pm 3.6$ & $71.6 \pm 3.1^{* \#}$ & $<0.001$ \\
\hline
\end{tabular}

Notes: Data are presented as mean \pm SD; Group B = bupivacaine group; Group FB = bupivacaine and fentanyl group; Group DB = bupivacaine and dexmedetomidine group; ObsQoR-11 = obstetric quality of recovery-11 score, $0-10$ in each term, where $0=$ strongly agree and $10=$ strongly disagree ${ }^{*} P<0.017$ Group DB or Group FB vs Group B; ${ }^{\#} P<0.017$ Group DB vs Group FB

\section{Discussion}

Our results showed that compared with $9 \mathrm{mg}$ of bupivacaine alone, the combination of $5 \mu \mathrm{g}$ of DEX for cesarean section could significantly prolong the duration of sensory block and improve paturients' recovery quality with no neonatal adverse effects or maternal neurotoxicity in the short term.

Spinal anesthesia, which is block-well, easy to operate, not as complicated as epidural anesthesia [19], and avoiding the maternal risk of general anesthesia, has become the preferred anesthesia type for cesarean section.
However, in clinical practice, single-shot spinal anesthesia was often not sufficient to inhibit visceral pain, causing maternal discomfort during the surgery, which affect parturients' postoperative recovery quality [6]. While increasing the doses of local anesthetics to prolong the analgesic time could lead to adverse effects such as central nervous system problems and cardiotoxicity. In our study, compared with intrathecal $9 \mathrm{mg}$ of bupivacaine alone, the onset time of sensory and motor block of parturients in combination of $9 \mathrm{mg}$ of intrathecal bupivacaine with $5 \mu \mathrm{g}$ of DEX was significantly

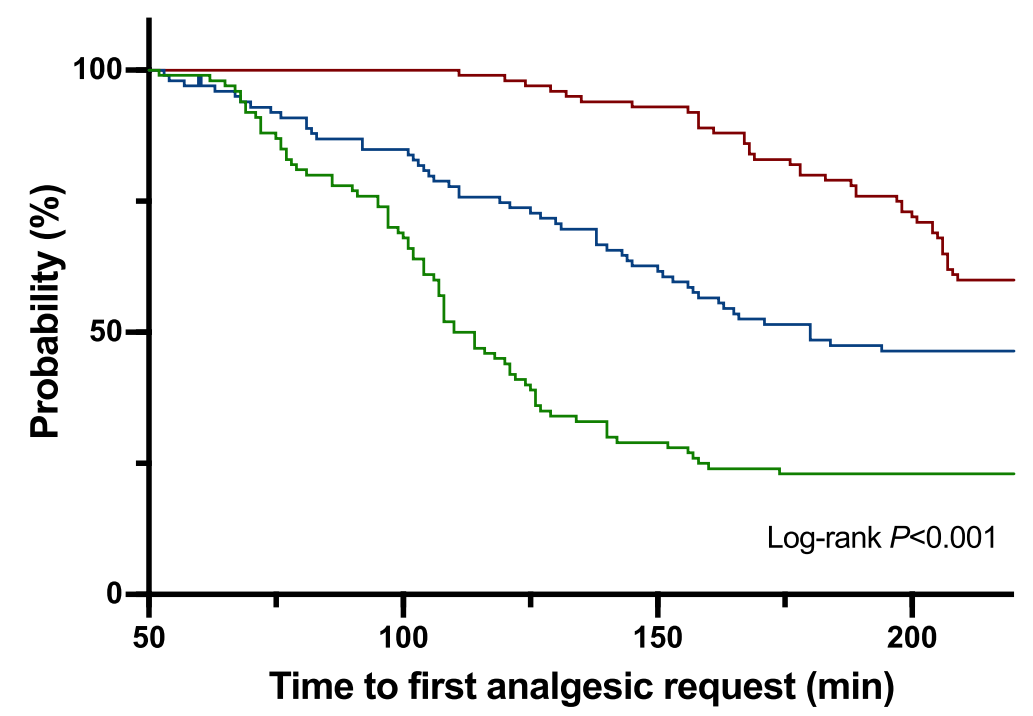

$\perp$ Group B $\rightarrow$ Group FB $\perp$ Group DB

Fig. 3 Kaplan-Meier curves for time to first analgesic request. Notes: Group B = bupivacaine group; Group FB= bupivacaine and fentanyl group; Group DB = bupivacaine and dexmedetomidine group 

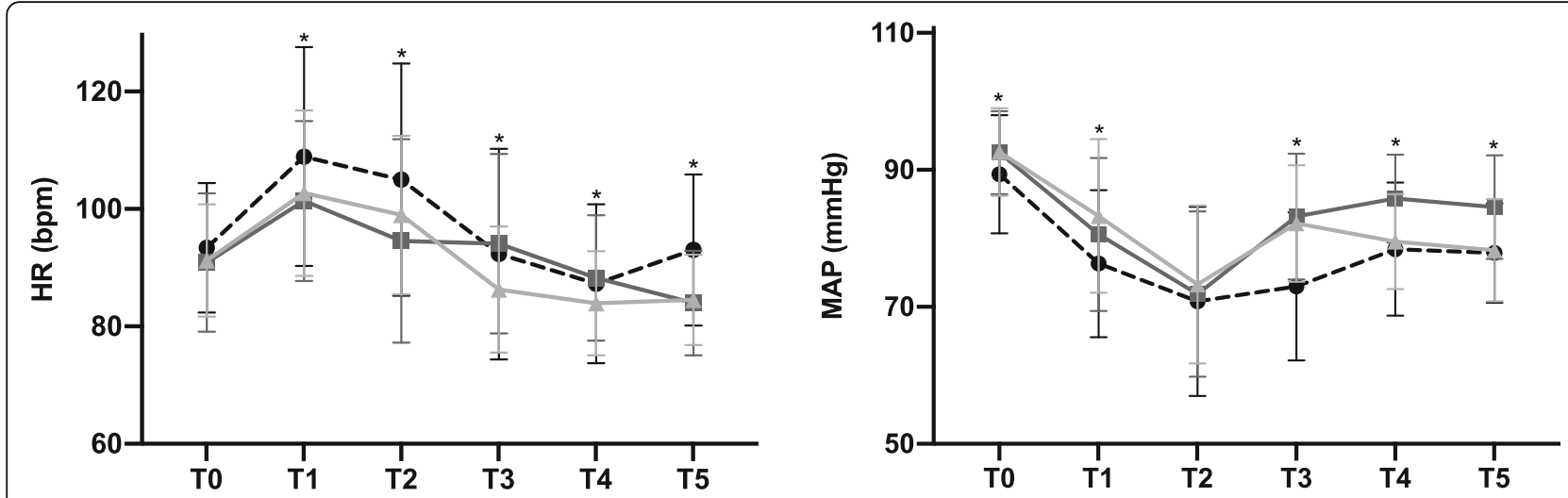

$\rightarrow$ Group B $\rightarrow$ Group FB $\leftarrow$ Group DB

Fig. 4 Hemodynamic parameters. Notes: Group B = bupivacaine group; Group FB = bupivacaine and fentanyl group; Group DB= bupivacaine and dexmedetomidine group; $\mathrm{T} 0$ = before spinal anesthesia, $\mathrm{T} 1, \mathrm{~T} 2, \mathrm{~T} 3, \mathrm{~T} 4, \mathrm{~T} 5=0,5,10,15,20 \mathrm{~min}$ after spinal anesthesia. $\mathrm{HR}=\mathrm{Heart}$ Rate; $\mathrm{MAP}=$ Mean Arterial Pressure; *There were significant differences among the three groups $(P<0.05)$

shortened, and the duration of sensory block was significantly prolonged by $40 \mathrm{~min}$, which is consistent with the research results of Suthar's [20] and Sushruta's [10]. The mechanism may be as follows: DEX can activate the $\alpha-2$ adrenergic receptor in the dorsal horn neurons, activate the spinal cord intermediate neurons by reducing the neurotransmitter released by the primary afferent end and G-protein-mediated potassium channel, and make the spinal cord intermediate neurons hyperpolarized, thus reducing the pain transmission. In addition, DEX can also block the internal flow of $\mathrm{Na}+$ and enhance the blocking effect of local anesthetics on the sodium channel of the cell membrane [21, 22]. However, consisted with the results of a meta-analysis [23] that included 9 RCTs, our study found that motor block duration of paturients with intrathecal DEX was also prolonged, which suggest that combination with DEX may increase the fall risk and delay the early rehabilitation of parturients.

Currently, the commonly used postoperative recovery quality scales were QoR-40 [24] and QoR-15 [25]. However, both of them are developed and verified in nonobstetric patients and day surgery population [26], so there are many items unrelated to cesarean section, and lack of critical elements to evaluate postoperative recovery after delivery such as the ability of caring babies [18]. The ObsQoR-11 scale has been proved to be reliable, clinically acceptable, feasible and effective in patients undergoing elective and emergency cesarean section [18, 27]. In our study, all questionnaire feedback had been received and the results showed that scores in group $\mathrm{DB}$ was higher than in both group FB and group $B(P<0.017)$, suggesting that parturients with intrathecal DEX had a better recovery quality.

Consistent with the findings of meta-analysis conducted by Miao [12], intrathecal DEX can significantly reduce the incidence of shivering in parturients undergoing spinal anesthesia. The mechanism of antishivering effect can be inferred that DEX could reduce central thermos-sensitivity by weakening the electrical conductivity of neurons through mediating the $\alpha-2$ adrenergic receptors in the brain and spinal cord [28, 29]. Moreover, intrathecal DEX showed added advantages on block characteristics and ObsQoR-11 score compared with intrathecal fentanyl, suggesting a better clinical application prospect.

Table 3 Maternal outcomes

\begin{tabular}{lllll}
\hline & Group B $(n=100)$ & Group FB $(n=100)$ & Group DB $(n=100)$ & $P$-Value \\
\hline Shivering, $\mathrm{n}(\%)$ & $35(35)$ & $18(82) *$ & $3(97) *^{* *}$ & $33(33)$ \\
Hypotension, $\mathrm{n}(\%)$ & $28(28)$ & $25(25)$ & $1.5 \pm 3.0$ & 0.450 \\
Dose of ephedrine $(\mathrm{mg})$ & $2.8 \pm 3.9$ & $2.1 \pm 3.6$ & $14(14)$ & 0.029 \\
Nausea and/or vomiting, $\mathrm{n}(\%)$ & $11(11)$ & $17(17)$ & $105.0 \pm 7.8$ & 0.474 \\
Sufentanil consumption $(\mu \mathrm{g})$ & $106.0 \pm 9.2$ & $105.5 \pm 7.7$ & 0.681 \\
\hline
\end{tabular}

Notes: Data are presented as $\mathrm{n}(\%)$ or mean \pm SD; Group $\mathrm{B}=$ bupivacaine group; Group FB = bupivacaine and fentanyl group; Group DB = bupivacaine and dexmedetomidine group

${ }^{*} P<0.017$ Group DB or Group FB vs Group B; ${ }^{\#} P<0.017$ Group DB vs Group FB 
Table 4 Neonatal outcomes

\begin{tabular}{|c|c|c|c|c|}
\hline & Group B $(n=100)$ & Group FB $(n=100)$ & Group DB $(n=100)$ & $P$-Value \\
\hline \multicolumn{5}{|l|}{ Umbilical artery } \\
\hline $\mathrm{pH}$ & $7.3 \pm 0.3$ & $7.3 \pm 0.2$ & $7.3 \pm 0.4$ & 0.581 \\
\hline $\mathrm{PaO}_{2}(\mathrm{mmHg})$ & $15.6 \pm 2.0$ & $15.0 \pm 2.3$ & $15.4 \pm 2.4$ & 0.217 \\
\hline $\mathrm{PaCO}_{2}(\mathrm{mmHg})$ & $49.9 \pm 3.4$ & $49.8 \pm 4.0$ & $50.4 \pm 4.6$ & 0.545 \\
\hline \multicolumn{5}{|l|}{ Umbilical vein } \\
\hline $\mathrm{pH}$ & $7.4 \pm 0.2$ & $7.4 \pm 0.1$ & $7.4 \pm 0.5$ & 0.711 \\
\hline $\mathrm{PaO}_{2}(\mathrm{mmHg})$ & $30.0 \pm 3.4$ & $30.7 \pm 4.4$ & $30.80 \pm 3.5$ & 0.277 \\
\hline $\mathrm{PaCO}_{2}(\mathrm{mmHg})$ & $42.6 \pm 3.2$ & $41.6 \pm 3.8$ & $42.0 \pm 3.2$ & 0.111 \\
\hline \multicolumn{5}{|l|}{ Apgar score } \\
\hline $1 \mathrm{~min}$ & $8.7 \pm 0.5$ & $8.7 \pm 0.5$ & $8.7 \pm 0.5$ & 0.752 \\
\hline $5 \mathrm{~min}$ & $9.7 \pm 0.5$ & $9.7 \pm 0.4$ & $9.7 \pm 0.5$ & 0.809 \\
\hline
\end{tabular}

Notes: Data are presented as mean \pm SD; Group B = bupivacaine group; Group FB = bupivacaine and fentanyl group; Group DB = bupivacaine and dexmedetomidine group

${ }^{*} P<0.017$ Group DB or Group FB vs Group B; ${ }^{\# P}<0.017$ Group DB vs Group FB

When intrathecal DEX during cesarean section, one of the main concerns was the maternal neurotoxicity. Therefore, all participants were followed up for 30 days after surgery, and none of them showed neurological complications of lower limbs and buttocks, indicating that intrathecal DEX would not lead to nerve injury in the short term. Ozdamar [30] injected 10 rats with DEX $10 \mu \mathrm{g}$ through the subarachnoid path and extracted spinal medulla for histological and electron microscopy examination after 7 days, and the results showed that compared with saline group, no signs of neuronal or axonal injury, gliosis, or myelin sheath damage was found. Another concern was the adverse effects on the fetus, which was excluded by the blood gas analysis and Apgar scores in our study. Li et al. [31] showed similar results, which further confirmed our conclusion. A placental perfusion study in vitro conducted by Ala-Kokko [32] found that the DEX fetal: maternal concentration ratio was 0.77 , which meant DEX in maternal circulation was easy to pass through the placental barrier. Currently, there is no firm clinical data about whether DEX would be absorbed into the maternal circulation and transferred to the fetus via the placenta under the intrathecal administration. In our study, the plasma concentration of DEX in the UA and UV was measured and no DEX accumulation was detected, suggesting that intrathecal $5 \mu \mathrm{g}$ of DEX caused a low or even no drug exposure on the fetus, which would not lead to adverse effects.

There were also some limitations in our study. Firstly, the adequacy of muscle relaxation during the surgery and the satisfaction of parturients and obstetricians were not measured, further studies should use more parameters to explore the efficacy of intrathecal DEX; Secondly, due to the invasive arterial blood pressure monitoring was not performed during the operation, the information concerning the placental transfer was not obtained with no maternal blood plasma sample collected; Thirdly, we did not investigate the dose-response reaction of DEX, and the optimal clinical dose was not determined. Furthermore, the postoperative follow-up period in this study was only 30 days, so it is unknown whether patients had delayed adverse neuron reactions.

\section{Conclusion}

DEX is a potential local anesthetic adjuvant that the intrathecal combination of $5 \mu \mathrm{g}$ DEX can safely exhibit a facilitatory block effect and improve parturients' recovery quality. However, large sample clinical studies to support the safety of intrathecal DEX use in the clinical setting are still needed.

\section{Supplementary information}

Supplementary information accompanies this paper at https://doi.org/10. 1186/s12871-020-01109-4

Additional file 1:. Table S1 Block characteristics.

Abbreviations

DEX: Dexmedetomidine; Cl: Confidence Interval;; SpO2: Pulse Oxygen Saturation; HR: Heart Rate; ECG: Electrocardiogram; NIBP: Noninvasive Blood Pressure; SBP: Systolic Blood Pressure; MBS: Modified Bromage scale; ObsQoR-11: Obstetric Quality of Recovery-11 Score; MAP: Mean Arterial Pressure; RR: Relative Risk; ASA: American Society of Anesthesiologists; BMI: Body Mass Index

Acknowledgments

This study thanked Ph.D. Yuan and his team for offering help in plasma concentration measurement of dexmedetomidine.

Authors' contributions

LXX contributed to study design, interpretation of data, and drafted the manuscript. LS contributed to study design, and interpretation of the data, revised the manuscript, and approved the final version. LYM was primarily responsible for the processing and analysis of blood samples. LXL contributed to analysis, and approved the final version. WXH was responsible for the follow up. All authors have read and approved the final manuscript. 


\section{Funding}

This study was supported by the Qing Lan Project of Jiangsu Province; the Nature Science Foundation of Jiangsu Province (BK20161175); the "Six One" Project of Jiangsu Province (LGY2016039); Natural Science Research Project of Jiangsu Higher Education Institutions (17KJA3320006).

\section{Availability of data and materials}

The datasets generated and/or analyzed during the current study are available from the corresponding author on reasonable request.

\section{Ethics approval and consent to participate}

This prospective, double-blind, randomized controlled trial was approved by the ethics committee of Feng Xian People's Hospital and the Affiliated Hospital of Xuzhou Medical University. Written informed consent was obtained from all participants.

\section{Consent for publication}

Not applicable.

\section{Competing interests}

The authors report no conflicts of interest in this work.

\section{Author details}

${ }^{1}$ Jiangsu Province Key Laboratory of Anesthesiology, Xuzhou Medical University, Xuzhou, Jiangsu, China. ${ }^{2}$ Feng Xian People's Hospital of Jiangsu Province, Xuzhou, Jiangsu, China. ${ }^{3}$ Department of Anesthesiology, the Affiliated Hospital of Xuzhou Medical University, 99 Huaihai West Road, Xuzhou 221000, Jiangsu, China.

Received: 14 May 2020 Accepted: 26 July 2020

Published online: 03 August 2020

\section{References}

1. Juang J, Gabriel RA, Dutton RP, et al. Choice of anesthesia for cesarean delivery: an analysis of the national anesthesia clinical outcomes registry. Anesth Analg. 2017:124:1914-7.

2. Kim WH, Hur M, Park SK, et al. Comparison between general, spinal, epidural, and combined spinal-epidural anesthesia for cesarean delivery: a network meta-analysis. Int J Obstet Anesth. 2019;37:5-15.

3. Ghaffari S, Dehghanpisheh $L$, Tavakkoli F, et al. The effect of spinal versus general anesthesia on quality of life in women undergoing cesarean delivery on maternal request. Cureus. 2018;11(12):e3715.

4. Riley ET. Regional anesthesia for cesarean section. Tech Reg Anesth Pain Manage. 2003;7:204-12.

5. Wu CL, Rowlingson AJ, Partin AW, et al. Correlation of postoperative pain to quality of recovery in the immediate postoperative period. Reg Anesth Pain Med. 2005;30(6):516-22.

6. Catro A, Lucas S, De A, et al. The effect of Neuraxial versus general anesthesia techniques on postoperative quality of recovery and analgesia after abdominal hysterectomy: a prospective randomized controlled trial. Anesth Analg. 2011;113(6):1480-6.

7. Fernandes HS, Bliacheriene F, Vago TM, et al. Clonidine effect on pain after cesarean delivery: a randomized controlled trial of different routes of administration. Anesth Analg. 2018;127(1):165-70.

8. Uppal V, Retter S, Casey M, et al. Efficacy of intrathecal fentanyl for cesarean delivery: a systematic review and meta-analysis of randomized controlled trials with trial sequential analysis. Anesth Analg. 2019;130(1):111.

9. Hall JE, Uhrich TD, Barney JA, et al. Sedative, amnestic, and analgesic properties of small-dose Dexmedetomidine infusions. Anesth Analg. 2000; 90(3):699-705

10. Sushruth MR, Rao DG. Effect of adding intrathecal dexmedetomidine as an adjuvant to hyperbaric bupivacaine for elective cesarean section. Anaesth Pain Intensive Care. 2018;22(3):348-54

11. Gupta M, Gupta P, Singh DK. Effect of 3 different doses of intrathecal dexmedetomidine $(2.5 \mathrm{mg}, 5 \mathrm{mg}$, and $10 \mathrm{mg}$ ) on subarachnoid block characteristics: a prospective randomized double-blind dose-response trial Pain Physician. 2016;19(3):e411-20.

12. Miao S, Shi M, Zou L, et al. Effect of intrathecal dexmedetomidine on preventing shivering in cesarean section after spinal anesthesia: a metaanalysis and trial sequential analysis. Drug Des Devel Ther. 2018;12:3775-83.
13. Singh AP, Chawla S, Bajwa SJS, et al. Efficacy and safety of dexmedetomidine as an intrathecal agent: a dose finding clinical study. Anaesth Pain Intensive Care. 2017;21(1):13-8

14. Axelsson K, Widman GB. A comparison of bupivacaine and tetracaine in spinal anaesthesia with special reference to motor block. Acta Anaesthesiol Scand. 1985;29:79-86.

15. Russell IF. Assessing the block for caesarean section. Int J Obstet Anesth. 2001;10(2):83-5.

16. Wang $X$, Fang F, Zhu XG. Clinical application of combined spinal-epidural anesthesia with fentanyl and ropivacaine in cesarean section. J Southeast Univ (Med Sci Edi). 2014:33(2):170-3.

17. Pais de Barros JP, Gautier T, Sali W, et al. Quantitative lipopolysaccharide analysis using HPLC/MS/MS and its combination with the limulus Amebocyte lysate assay. J Lipid Res. 2015;56(7):1363-9.

18. Ciechanowicz S, Setty T, Robson E, et al. Development and evaluation of an obstetric quality-of-recovery score (ObsQoR-11) after elective caesarean delivery. Br J Anaesth. 2019:122(1):69-78.

19. Heesen M, Klöhr S, Rossaint $R$, et al. Insertion of an intrathecal catheter following accidental dural puncture: a meta-analysis. Int J Obstet Anesth. 2013:22(1):26-30.

20. Suthar $O$, Sethi $P$, Sharma UD. A comparison of intrathecal dexmedetomidine, clonidine, and fentanyl as adjuvants to hyperbaric bupivacaine for lower limb surgery: a double-blind controlled study. $J$ Anaesthesiol Clin Pharmacol. 2013:29(4):496-502

21. Khan ZP, Ferguson CN, Jones RM. Alpha-2 and imidazoline receptor agonists. Their pharmacology and therapeutic role. Anaesthesia. 1999;54(2): $146-65$

22. Oda A, lida $\mathrm{H}$, Tanahashi $\mathrm{S}$, et al. Effects of alpha2-adrenoceptor agonists on tetrodotoxin-resistant $\mathrm{Na}+$ channels in rat dorsal root ganglion neurons. Eur J Anaesthesiol. 2007;24(11):934-41.

23. Abdallah FW, Brull R. Facilitatory effects of perineural dexmedetomidine on neuraxial and peripheral nerve block: a systematic review and meta-analysis. $\mathrm{Br} J$ Anaesth. 2013:110(6):915-25.

24. Myles PS, Weitkamp B, Jones $K$, et al. Validity and reliability of a postoperative quality of recovery score: the QoR-40. Br J Anaesth. 2000;84: 11 e5

25. Stark PA, Myles PS, Burke JA. Development and psychometric evaluation of a postoperative quality of recovery score: the QoR-15. Anesthesiology. 2013; 118:1332e40.

26. Chazapis $M$, Walker EM, Rooms MA, et al. Measuring quality of recovery-15 after day-case surgery. Br J Anaesth. 2016;116:241e8.

27. Ciechanowicz S, Howle R, Heppolette C, et al. Evaluation of the obstetric quality-of-recovery score (ObsQoR-11) following non-elective caesarean delivery. Int J Obstet Anesth. 2019:39:51-9.

28. Nasseri K, Ghadami N, Nouri B. Effects of intrathecal dexmedetomidine on shivering after spinal anesthesia for cesarean section: a double-blind randomized clinical trial. Drug Des Devel Ther. 2017;11:1107-13.

29. Mittal G, Gupta K, Katyal S, et al. Randomised double-blind comparative study of dexmedetomidine and tramadol for post-spinal anaesthesia shivering. Indian J Anaesth. 2014;58:257-62.

30. Ozdamar D, Dayioglu H, Anik I, et al. Evaluation of the neurotoxicity of intrathecal dexmedetomidine on rat spinal cord (electro microscopic observations). Saudi J Anaesth. 2018;12(1):10-5.

31. Li Z, Tian M, Zhang CY, et al. A randomized controlled trial to evaluate the effectiveness of intrathecal bupivacaine combined with different adjuvants (fentanyl, clonidine and Dexmedetomidine) in caesarean section. Drug Res. 2015;65(11):581-6.

32. Ala-Kokko TI, Pienimäki P, Lampela E, Hollmén Al, Pelkonen $\mathrm{O}$, Vähäkangas K. Transfer of clonidine and dexmedetomidine across the isolated perfused human placenta. Acta Anaesthesiol Scand. 1997;41(2):313-9.

\section{Publisher's Note}

Springer Nature remains neutral with regard to jurisdictional claims in published maps and institutional affiliations. 